\title{
Sebaceous Carcinoma and Muir - Torre Syndrome: Case Report
}

\section{Louis Smits $^{1 *}$, Sorin Cimpean ${ }^{2}$ and Aude Schillaci ${ }^{3}$}

${ }^{1}$ Assistant UCL, Digestive Surgery, Notre Dame de Grâce Clinic, Gosselies, Belgium

${ }^{2}$ Digestive Surgery, Notre Dame de Grâce Clinic, Gosselies and Digestive Surgery, Saint Pierre University Hospital, Brussels, Belgium

${ }^{3}$ Digestive Surgery, Notre Dame de Grâce Clinic, Gosselies, Belgium

*Corresponding Author: Louis Smits, Assistant UCL, Digestive Surgery,

Notre Dame de Grâce Clinic, Gosselies, Belgium.
Received: February 18, 2020

Published: March 30, 2020

(C) All rights are reserved by Louis Smits., et

al.

\section{Abstract}

Muir-Torre Syndrome is a rare autosomal dominant genodermatosis characterized by the presence of cutaneous tumors, sebaceous adenoma or sebaceous carcinoma type, with or without keratoacanthoma, associated synchronously or metachronically with deep tumors of the same spectrum as Lynch syndrome.

We present here the case of a 47-year-old patient, in whom a MTS was detected following the discovery of a cutaneous lesion of seborrheic carcinoma type.

The discovery of cutaneous lesions of sebaceous adenoma, sebaceous carcinoma or keratoacanthoma coupled with a family history of neoplasia must make suspect a MTS. Since this syndrome is autosomal dominant, family screening should be offered to all members of the family at risk. The follow-up of patients with MTS is essential for life given the risk of cutaneous and /or deep tumor recurrence.

Keywords: Lynch Syndrome; Sebaceous Tumor; Sebaceous Carcinoma

\section{Abbreviations}

DNA: Acid Deoxyribonucleic; MMR: Mismatch Repair; MSI: Microsatellite Instability; MTS: Muir-Torre Syndrome.

\section{Introduction}

Sebaceous cysts are common in the general population. The presence of adenoma or sebaceous carcinoma is much less so. MTS is an autosomal dominant genodermatosis described in the literature as a phenotypic variant of Lynch syndrome. These syndromes have great similarities from a bio-molecular point of view, especially with the presence, in the majority of cases, of MSI resulting from an inactivating germline mutation in one of the genes of the MMR system responsible for the repair of DNA mismatches.

Clinically, MTS is characterized by the presence of cutaneous tumors of the adenoma or sebaceous carcinoma type, with or with- out keratoacanthoma, synchronously or metachronically associated with deep tumors of the same spectrum as Lynch syndrome.

We report the case of a 47-year-old patient diagnosed with MTS following the discovery of a cutaneous lesion of seborrheic carcinoma type.

\section{Case Report}

A 47-year-old patient with medical history of right nephrectomy in 2012 for a clear cell renal adenocarcinoma (stage pT1a) was referred to the surgical consultation for an infected cutaneous mass who presented a partially necrosis located on right infra-clavicular area who appeared two months ago (Figure 1). The first hypothesis of diagnosis was of an infected sebaceous cyst. The mass was resected in one piece and then sent for anatomopathological analysis.

Citation: Louis Smits., et al. "Sebaceous Carcinoma and Muir - Torre Syndrome: Case Report”. Acta Scientific Gastrointestinal Disorders 3.4 (2020): 06-08. 


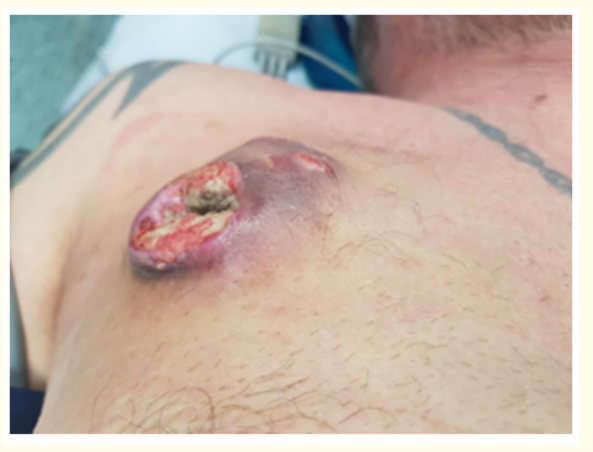

Figure 1: Preoperative image.

This analysis revealed a highly moderately ulcerated neoplastic lesion of moderately differentiated sebaceous carcinoma (grade 2) of $4 \mathrm{~cm}$ long axis. No image of angiolymphatic permeation is observed. It is therefore a stage pT2. Immunohistochemical and molecular analyzes were also performed. This revealed microsatellite instability with loss of MSH2 expression (MMR).

An additional assessment was carried out considering the suspicion of an MTS. The medical family history revealed that the patient's father died at age 62 from colon cancer, his mother died at age 31 from bone cancer and his grandfather also died of unspecified cancer.

The PET-scan revealed two suspicious colic lesions. A colonoscopy was performed and revealed two polyps that were resected. The analysis of these revealed an adenomatous polyp in low grade dysplasia and another one with a moderately differentiated adenocarcinoma. Immunohistochemical analysis of the polyps revealed MSI with MSH2 loss of expression similar to that observed in the cutaneous lesion. Other cutaneous lesions were discovered and were resected. Among them, four lesions of the epidermal cyst type, two lesions of sebaceous hyperplasia type and a sebaceous adenoma type lesion. Subsequently, genetic analyses were requested and showed gene mutation MSH2 (c.1147C>T).

The diagnosis of MTS was retained. Regular dermatological follow-up to monitor the evolution of the cutaneous lesions and gastroenterological follow up are needed. Genetic assessment is proposed for the patient's children for early detection of the syndrome.

\section{Discussion}

MTS is a rare genodermatosis with autosomal dominant inheritance described in 1967 by Muir E, Bell A and Barlow K [1]. This syndrome is characterized by the presence of at least one sebaceous cutaneous tumor (type adenoma or carcinoma) with or without keratoacanthoma associated with at least one synchronous or metachronous visceral tumor, most often colorectal and usually with low grade of malignancy [1-3].

The literature distinguishes two types of MTS: The first type (70\% of cases), as in our case study, is considered a variant of Lynch syndrome and shows instability of microsatellites with loss of expression and mutation in MMRs [3-5]. These proteins are responsible for the detection and reparation of the errors during DNA replication. The most common MMR mutation in MTS is MSH2 (over $90 \%$ of cases) $[3,5]$ as well as MLH1 (10\% of cases) and other rare mutations such as MSH6, PMS22 [6]. The second type of MTS does not present microsatellite instability. In this case, other mutations lead to a phenotype of MTS $[3,4,6]$.

MTS is found in $9.2 \%$ of patients with Lynch Syndrome. It mainly affects men (male to female ratio 3/2) [6] and the family history of cancers are to be taken into account in the diagnostic procedure.

Patients suffering from an MTS preferentially develop cutaneous tumors of sebaceous adenoma, sebocarcinoma or keratoacanthomas type as well as predominantly deep colorectal tumors [6-8]. Although sebaceous gland cysts are common in the general population, sebaceous adenomas, sebaceous carcinomas and epitheliomas are rarer and their discovery should be suggestive of MTS [4].

On the other hand, it appears that the sebaceous adenoma is found most frequently in the MTS (68\% of cases), followed by epitheliomas (25\% of cases) and sebaceous carcinomas $(30 \%$ of cases) [4,5]. Moreover, sebaceous carcinomas can also have local and metastatic aggressiveness. Their resection requires healthy margins of 5 to $6 \mathrm{~mm}[4]$.

Skin tumors in MTS syndrome are often multiple and recurrent. If they are preferably on the head or neck [3], they can also reach the limbs or the trunk [6] as with our patient. If these cutaneous lesions can be associated with other deep tumors, mainly colic [2], other cancers are also possible such as that of the small intestine 
[6], the duodenum, the rectum, the endometrium, the urothelium, the bile ducts, the ovaries [5].

The occurrence of skin lesions compared to the first deep lesions is variable. These may precede them (22\% of cases), be concomitant ( $6 \%$ of cases) or still occur after the discovery of the first deep tumors (56\% of cases) $[2,5,9]$. These carcinomas are characterized by a younger age of onset and are less aggressive. Their prognosis would be better than that of sporadic tumors [5].

Most cutaneous lesions of MTS seborrheic carcinoma are treated by complete excision.

Anatomopathological examination of the resected parts is fundamental to ensure healthy margins as well as the immunohistochemical examination allowing the search for MMR defect and a microsatellite instability.

In view of the clinic, regular monitoring will be recommended both dermatologically and gastrologically as we recommend to our patient and a regular gynecological examination will be proposed in women as in the Lynch syndrome.

Moreover, aspirin could be prescribed to the patient with MTS. Aspirin could reduce the risk of colorectal cancer. However, the optimal dose and duration should be still defined and are still the subject of the Cancer Prevention Programme current study [10].

\section{Conclusion}

The discovery of cutaneous lesions of sebaceous adenoma type, sebaceous carcinoma or keratoacanthoma coupled with a family history of neoplasia must make suspect an MTS or a Lynch syndrome and involve the search for possible visceral lesions and mutation of MMR genes and an instability of microsatellites. On the other hand, as MTS is autosomal dominant, family screening should be offered to all members of the family at risk to allow early detection of the syndrome and appropriate close follow-up in case of transmission of the syndrome in question. The follow-up of patients with MTS is essential for life given the risk of cutaneous and/ or deep tumor recurrence.

\section{Bibliography}

1. Muir E., et al. "Multiple primary carcinoma of the colon duodenum and larynx associated with kerato-acanthoma of the face". British Journal of Surgery 54 (1967): 191.
2. Pancholi A., et al. "Muir-Torre Syndrome: A case Report and Screening Recommendations". Gillingham, Kent: Annals of The Royal College of Surgeons of England (2008).

3. Ponti G., et al. "Identification of Muir-Torre Syndrome among Patients with sebaceous Tumors and Keratoacanthomas, Role of Clinical Features, Microsatellite instability and Immunohistochemistry". Wiley InterScience] Modena, Italy: American cancer Society (2005).

4. John AM and Schwartz RA. "Muir-Torre syndrome (MTS): An update and approach to diagnosis and management". Newark, New Jersey: American Academy of Dermatology (2015).

5. Ponti G., et al. "Muir-Torre Syndrome and founder mismatch repair gene mutations: A long gone historical genetic challenge". Modena, Italy: Elsevier B.V. (2015).

6. Bacha D., et al. "Syndrome de Muir-Torre: association rare à un carcinome duodenal". Annales de Pathologie. Clichy, France : Elsevier Masson (2009).

7. Le S., et al. "Lynch Syndrome and Muir-Torre Syndrome: An update and review on the genetics, epidemiology and management of two related disorders". Norfolk, Virginia: Dermatology Online Journal Review 23.11 (2017): 2.

8. Adan F., et al. "A squamous cell carcinoma in a young woman with Lynch syndrome". [Springer] Amsterdam, The Netherlands: Familial Cancer (2019).

9. Descamps V., et al. "Syndrome de Muir-Torre, réunion de concertation pluridisciplinaire". Paris, France : Images en Dermatologie, Janvier-février 7.1 (2014).

10. Burn J., et al. "Long-term effect of aspirin on cancer risk in carriers of hereditary colorectal cancer: an analysis from the CAPP2 randomised controlled trial". [378:2081-87] Newcastle, UK: Lancet (2011).

\section{Assets from publication with us}

- Prompt Acknowledgement after receiving the article

- Thorough Double blinded peer review

- Rapid Publication

- Issue of Publication Certificate

- High visibility of your Published work

Website: www.actascientific.com

Submit Article: www.actascientific.com/submission.php

Email us: editor@actascientific.com

Contact us: +919182824667 